\title{
Understanding biomolecular motion, recognition, and allostery by use of conformational ensembles
}

\author{
R. Bryn Fenwick • Santi Esteban-Martín • \\ Xavier Salvatella
}

Received: 24 April 2011/Revised: 6 September 2011/Accepted: 14 September 2011/Published online: 17 November 2011

(C) The Author(s) 2011. This article is published with open access at Springerlink.com

\begin{abstract}
We review the role conformational ensembles can play in the analysis of biomolecular dynamics, molecular recognition, and allostery. We introduce currently available methods for generating ensembles of biomolecules and illustrate their application with relevant examples from the literature. We show how, for binding, conformational ensembles provide a way of distinguishing the competing models of induced fit and conformational selection. For allostery we review the classic models and show how conformational ensembles can play a role in unravelling the intricate pathways of communication that enable allostery to occur. Finally, we discuss the limitations of conformational ensembles and highlight some potential applications for the future.
\end{abstract}

Keywords Dynamic ensembles - NMR .

Conformational selection · Induced fit · Allostery

Molecular recognition is of paramount importance in biology — without it life would not exist. Before the first 3D structures of biomolecules were determined (Watson and Crick 1953; Kendrew et al. 1958; Muirhead and Perutz 1963), the lock and key model of molecular recognition in

Special Issue: Transient interactions in biology.

R. B. Fenwick · S. Esteban-Martín · X. Salvatella $(\bowtie)$ Joint BSC-IRB Research Programme in Computational Biology, Institute for Research in Biomedicine, IRB Barcelona, Baldiri Reixac 10, 08028 Barcelona, Spain e-mail: xavier.salvatella@irbbarcelona.org

X. Salvatella Institució Catalana de Recerca i Estudis Avançats, ICREA, Barcelona, Spain the binding events associated with enzymatic catalysis had already been proposed (Fischer 1894). Over time, an appreciation of the structural changes that can occur upon binding led to the related induced fit (Koshland 1958) and fluctuation fit (Straub and Szabolcsi 1964) models. At about the same time two complementary models for describing allostery, a key biological mechanism that is responsible for information transfer, were also proposed, the concerted model (Monod et al. 1965) and the sequential model (Koshland et al. 1966). These models were proposed before the development of molecular dynamics (MD) simulations (McCammon et al. 1977), a method that enables atomic-level understanding of the types, amplitudes, and timescales of the motion of macromolecules and has influenced current views of how both molecular recognition and allostery occur (Karplus 2003; van Gunsteren and Dolenc 2008).

Molecular dynamics has provided significant insight into the details of molecular motion, but the significant contributions from experimental techniques cannot be overlooked. Indeed experiments provide direct evidence for dynamic processes, often at an atomic level, and can be used to validate the predictions of MD. Nuclear magnetic resonance (NMR) spectroscopy is a particularly powerful tool in the experimental analysis of macromolecular dynamics, because it furnishes information about both structure and motion at atomic resolution. This can be analysed by use of MD to generate conformational ensembles that enumerate the conformations adopted by a given macromolecule (Torda et al. 1989; Bonvin et al. 1994; Hess and Scheek 2003; Best and Vendruscolo 2004; Clore and Schwieters 2004b, 2006; Lindorff-Larsen et al. 2005a). The methods used to determine such ensembles have matured over the last 10 years to render ensemble refinement a leading approach to the characterisation of 
biomolecular motion (Vogeli et al. 2008; De Simone et al. 2009). These methods have recently been used to study molecular recognition and allostery and have provided new insights into their underlying mechanisms that are, importantly, supported by experimental results (Lange et al. 2008; Fenwick et al. 2011). To illustrate the types of representation of structural heterogeneity that can be obtained by use of NMR in combination with MD, in Fig. 1a we compare the results from these methods with those obtained by use of conventional tools for structure determination.

In addition to being important for fundamental reasons, theoretical or hybrid theoretical-experimental methods for characterisation of structural heterogeneity can be very important in structure-based drug discovery. In the near future, as recently demonstrated by the Al-Hashimi laboratory (Stelzer et al. 2011), it will indeed be possible to improve in-silico drug screening by use of conformational ensembles, because these contain the inherent functional motion of the therapeutic target. Here we review the advances that have been made in the understanding of the motion, molecular recognition, and allostery of biomolecules by use of conformational ensembles and discuss how these powerful techniques will continue to guide our understanding of these and related important biological phenomena.

\section{Biomolecular dynamics}

Given that molecular motion undoubtedly occurs under physiological conditions it is perhaps not surprising that functional roles have been attributed to it. Henzler-Wildman and Kern (2007) have recently reviewed this subject, emphasising the importance of motion for protein function. Examples include cytoskeletal function, antibody-antigen recognition, small molecule signalling, and information storage. Coarse-grained MD simulations have, for example, shown that the motion of actin filaments is important in
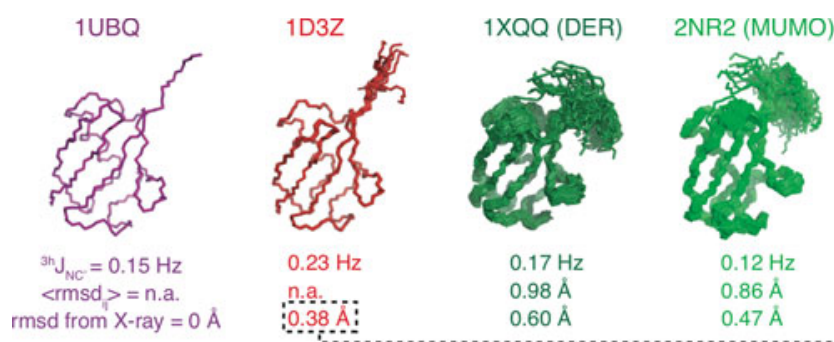

Fig. 1 Structures and ensembles of ubiquitin showing the ability of ensemble approaches to capture structural heterogeneity. $1 U B Q$ and $1 D 3 Z$ are the X-ray crystallography structure and the NMR average solution structure (purple and red), respectively. In green are two ensembles of motion on the sub- $\tau_{\mathrm{c}}$ timescale ( $<\sim 4$ ns for ubiquitin)
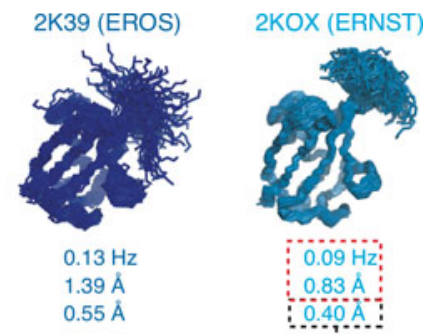

dictating the structural and functional properties of the cytoskeleton (Chu and Voth 2005) and, similarly, twodimensional correlation Fourier Transform Infrared (FTIR) spectroscopy has shown that structural flexibility is an inherent property of immunoglobulins (Kamerzell and Middaugh 2007); such flexibility is required for function because monoclonal IgG molecules must recognize antigens of various shapes and sizes. In the binding of a signalling molecule, GTP, by signal recognition particle (SRP) GTPase domains (Ramirez et al. 2008) it was hypothesized that flexibility enabled the regulation of GTPase activity by the cognate SRP receptor. Finally, a role of motion in the function of DNA has also been proposed (Blagoev et al. 2006) from MD simulations. In Fig. 2, we detail the timescales of motion for biomolecules and some experimental techniques that can, in principle, be used to study them. Motion of biological interest is often challenging to study because it occurs on timescales (ps to ms) that are accessible to a limited number of techniques such as NMR and dielectric relaxation spectroscopy (Fig. 2).

Although it is well-established, both from theoretical predictions and experimental observations, that macromolecules are highly dynamic at physiological temperatures, characterization of the motion is very challenging. The earliest MD simulation of a protein was of the small protein bovine pancreatic trypsin inhibitor (BPTI) (McCammon et al. 1977). McCammon et al. observed that atom displacements were substantial but that the resulting motion was correlated to conserve the structure of the protein. Increases in computer power have enabled study of the motion of systems of much larger size (Jensen et al. 2010) and on much longer timescales by use of these methods (Shaw et al. 2010). In Fig. 3, we highlight the work of D. E. Shaw Research, which observed slow motion, occurring on timescales longer than the rotational diffusion correlation time, of the backbone of a globular protein. This work shows that the motion of the backbone of BPTI occurs on longer timescales than that of the side chains, and suggests this is a general property of proteins.

and in blue are two ensembles that capture supra- $\tau_{\mathrm{c}}$ timescales up to ms. Below the structures is the agreement, in $\mathrm{Hz}$, with experimental hydrogen bond scalar couplings that are sensitive to a molecule's motion (small numbers are better), $\left\langle\operatorname{rmsd}_{i j}\right\rangle$, a measure of structural heterogeneity, and the RMSD from the X-ray crystal structure 


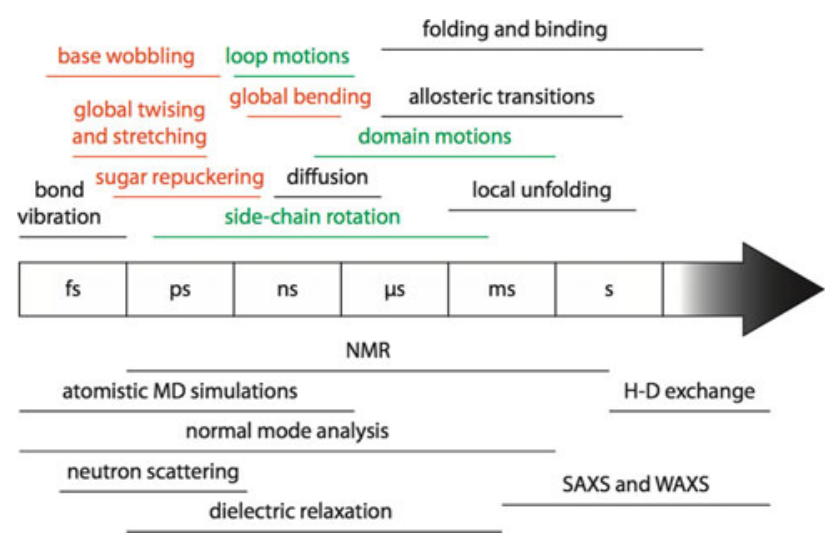

Fig. 2 Timescales of biological motion (above) and experimental and theoretical methods (below). Protein and nucleic acid dynamic timescales are shown in green and red, respectively. Timescales common to all biomolecules are shown in black. Experimental methods like smallangle X-ray scattering (SAXS) and wide-angle X-ray scattering (WAXS) are shown over the range they can detect fluctuations. Motion on faster timescales averages during the experiments

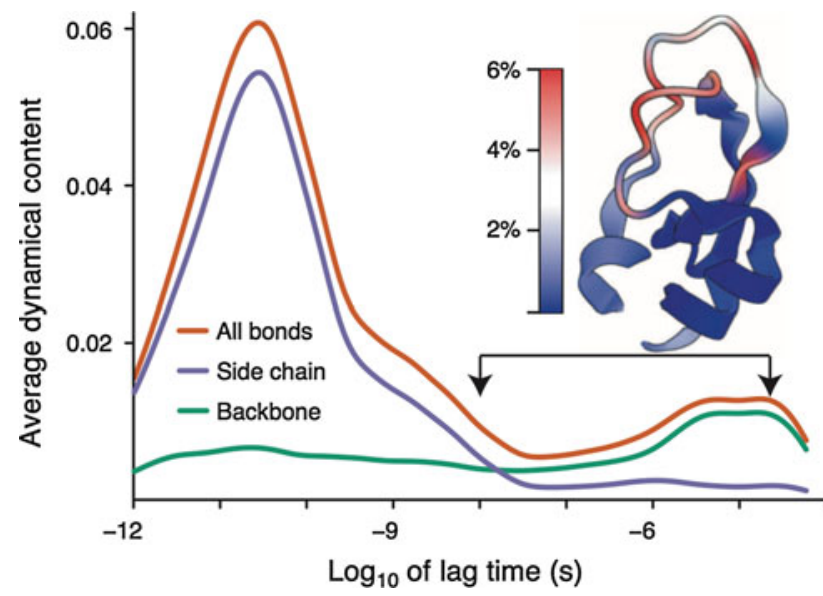

Fig. 3 Dynamic content of BPTI from a $1 \mathrm{~ms}$ simulation run by D. E. Shaw Research, showing that motion of side chains is pronounced on the sub- $\tau_{\mathrm{c}}$ timescale and that backbone motion is significant on the supra- $\tau_{c}$ timescale. Taken from Shaw et al. (2010)

Normal mode analysis, a theoretical tool that is complementary to $\mathrm{MD}$, and its coarse-grained equivalents, the Gaussian network model (Bahar et al. 2010) and the anisotropic network model (Zheng 2010), have been useful to determine the motion that occurs on potentially slower time scales (low-frequency modes). Normal mode analysis produces projections of the modes rather than conformational ensembles but can, in principle, probe longer timescales. Although relating the frequencies of motion of biomolecules to biological function has remained contentious (Kamerlin and Warshel 2010; Karplus 2010) the motion of biomolecules is likely to be related to their function.

The experimental evidence for protein motion comes from a wide range of techniques covering different timescales. Faure et al. (1994) were able to directly model the motion of lysozyme in the crystalline form by using atomic fluctuations around the mean atomic positions that give rise to diffuse scattering of the beam in diffraction experiments. Neutron scattering has also been used, in a similar manner, to detect motion in myoglobin (Kneller and Smith 1994; Frauenfelder and Mezei 2010), as has Mossbauer spectroscopy, by use of which it was observed that fluctuations of the solvent cause internal protein motion (Frauenfelder et al. 2009). Further evidence of motion from X-ray crystallography data comes in the form of the multiple conformations of proteins and nucleic acids that are obtained when the crystallization process is carried out several times. These conventional experiments can be complemented by single-molecule techniques that enable the observation of single states of a given molecule. Single molecule fluorescence energy transfer (FRET) spectroscopy, for example, enables, in principle, observation of distance distributions rather than average distances. These methods have provided very solid evidence that, because of macromolecular dynamics, inter-atomic distances are not fixed, either in proteins (Deniz et al. 2000) or in nucleic acids (Deniz et al. 1999).

The most detailed and exhaustive experimental studies of protein motion have been conducted with NMR spectroscopy. This technique has the ability to probe structural motion with atomic detail over the entire range of timescales from picoseconds to seconds (Fig. 2). Since the first comprehensive study of fast protein motion by NMR (Allerhand et al. 1971) it is has become routine to characterize the motion of proteins (Kay et al. 1989) that is faster than rotational diffusion by use of heteronuclear relaxation rates. For a recent review of the application of NMR to the study of the rapid motion of biomolecules and their complexes, the reader is directed to Jarymowycz and Stone (2006). In addition, Kay et al. have shown that it is possible to use the Carr-Purcell-Meiboom-Gill (CPMG) NMR measurements developed by Palmer et al. (2001) and Loria et al. (1999) to identify and characterize conformations that are present with a very low population (Korzhnev et al. 2010) when they are in relatively slow ( $\mu \mathrm{s}-\mathrm{ms})$ exchange with the most stable conformation of the macromolecule (Mittermaier and Kay 2006). Structural fluctuations occurring on the nanosecond to microsecond timescale can be probed by measurement of residual dipolar coupling (RDC) (Salmon et al. 2011).

\section{Biomolecular dynamics from ensembles}

Because the ability to visualize motion in macromolecules can provide details on how this contributes to biological function, a number of techniques have been developed for this purpose. Below are presented three methods that 
combine molecular simulation methods with the information provided by experimental techniques such as NMR; namely MD, MD selection methods, and restrained MD (Fig. 4). We concentrate mainly on the application of such methods and the insights they have provided rather than addressing the underlying principles.

Since the first simulations of biomolecules MD has become the most common method for studying the motion of proteins and nucleic acids (Karplus 2003). Simulations are started from a known configuration of the molecule derived from experimental (X-ray or NMR) data or determined by homology modelling. Use of the ensembles generated from MD to analyse the function of motion for proteins and nucleic acids is too extensive to discuss in detail here; we therefore highlight some of the newer applications. In conventional MD, the starting configuration evolves in discrete time steps to produce a trajectory that simulates the fate of the molecule under a force field that models the internal energy of the system as the sum of simple potentials (Fig. 4a). In selection methods the agreement of such trajectories with experiment is improved by reassigning the statistical weights of the snapshots of the trajectory (Fig. 4b). Structure calculations using experimental data often restrain the structure at each step, as is shown in Fig. 4c, and do not report on the structural heterogeneity caused by dynamics. Time and ensemble restraining schemes in Fig. $4 \mathrm{~d}$, e can, instead, lead to ensembles that accurately capture the structural heterogeneity of the system as reflected by the experimental data.

The motion of bacteriophage T4 lysozyme (B4L) and adenylate kinase (AdK) has been studied by MD for a number of years. B4L is a hydrolytic enzyme composed of two domains and its dominant motion changes the orientation between them. In the crystal the protein is in a closed conformation but MD studies indicate that in solution it is likely to be more open than the crystal structures suggest (de Groot et al. 1998). This open state of the molecule has also been observed experimentally (Goto et al. 2001) and it is likely that this protein passes between the open and closed states via collective opening of the active site (Hub and de Groot 2009). One question that remains unanswered, however, is what the populations of the open and closed states are for this molecule. For AdK, it is well known that motion is highly important in biological function (Olsson and Wolf-Watz 2010). This protein is known to undergo large conformational changes in both the presence and absence of substrates in solution (Beckstein et al. 2009). It seems that the binding and release of substrate, which are rate-limiting for this enzyme, are related to these conformational changes. The link between catalysis and the structural changes that this protein undergoes is however hotly debated.

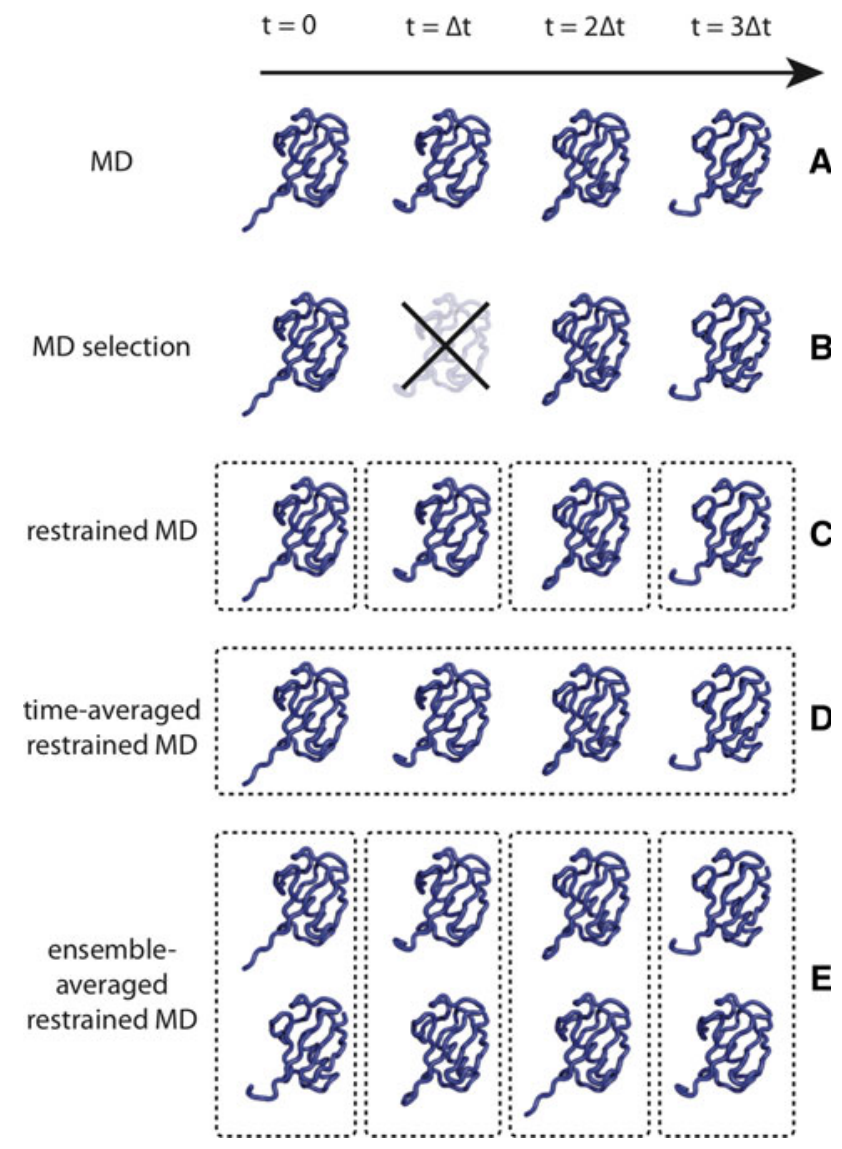

Fig. 4 Atomistic MD unrestrained and restrained schemes. For the restrained simulations, averaging of the experimental data is indicated as dashed lines. For unrestrained simulations, no restraints are present and the final ensemble is the sum of all frames (a). In MD selection no restraints are applied during the simulation, the final ensemble is selected on the basis of experimental data, eliminating structures that reduce the fit with experimental data (b). Restrained MD enforces the experimental data at each time point and, as a result, each structure is considered a different model of the average structure (c). During time-averaged restrained MD (d) a memory function biases the simulation to fulfil the restrained data over a given time period, the final ensemble samples the timescale up to the length of the memory function. Ensemble-averaged restrained MD restrains the experimental data at each step as in restrained MD, however the average runs over multiple parallel molecules that react to fulfil the experimental data at each time point over the ensemble (e). The final ensemble is a single snapshot of the parallel trajectories, and the timescale of the average is limited only by the timescale of the experimental data

Shaw et al. (2010) have recently shown the power of MD simulations by developing specific hardware and software for this purpose. By running microsecond-long MD simulations of the Kc1.2 pore domain they were able to show that potassium channel gating is a result of solvent expulsion from the active site, and confirmed the previously predicted model of ion transport (Hodgkin and Keynes 1955). Simulations of DNA and RNA are more limited, because of the relatively lower quality of the force 
fields, but are now maturing (Perez et al. 2007b), making it possible to explore the functional motion of nucleic acids. Comparison of the motion of double-stranded DNA and RNA, for example, has recently shown that RNA duplexes are more rigid than their DNA counterparts (Perez et al. 2007a; Faustino et al. 2010). It is well-established that MD suffers from limitations (van Gunsteren and Dolenc 2008) but, despite this, MD descriptions of macromolecular motion are quickly increasing in quality and this technique will continue to be a useful approach to determining ensembles.

As previously mentioned, when experimental data are available it is possible to use this information to prune the structures from an MD trajectory. This approach, which reweights the frames of an MD trajectory so that the trajectory is collectively consistent with experimental data (Fig. 4b), was used in an analysis of the motion of five globular proteins (Chen et al. 2007) by use of an NMR order parameter $\left(S^{2}\right)$ that reports on motion that is faster than the rotational diffusion of the macromolecule. This variable takes values between zero and unity, where zero represents unrestricted motion of the bond vector in the molecular frame and a value of unity indicates the vector is rigid. In Table 1, we show results from a selection method applied to five different proteins. The selection method was used to generate ensembles in good agreement with the experimental data, even when the underlying MD trajectories were not. The selection method was also used to great effect by Frank et al. (2009) to produce ensembles reporting on RNA motion; the structures of the conformational ensemble showed twisting of the individual RNA helices relative to one another in solution (Frank et al. 2009). A trajectory reweighing method has recently been proposed to improve MD force fields, but the approach can also be used to generate an ensemble from which functional motion can be extracted (Li and Brüschweiler 2010; Long and Brüschweiler 2011a).

Time-averaged and ensemble-averaged ensembles can be seen as equivalent although they are generated using MD by using different approaches. In restrained timeaveraged MD, which has been used to great effect and is

Table 1 Correlation with the experimental backbone order parameters $\left(S^{2}\right)$ for unrestrained MD and for the selection method (SAS)

\begin{tabular}{lll}
\hline Protein name & $\begin{array}{l}\text { Correlation for } \\
\text { unrestrained MD }\end{array}$ & Correlation for SAS \\
\hline Eglin c & $0.3-0.5$ & $0.96-0.98$ \\
Ubiquitin & $0.6-0.8$ & 0.99 \\
TNfn3 & $0.2-0.5$ & $0.98-0.99$ \\
$\beta$ ARK1 PH domain & $0.6-0.8$ & $0.97-0.98$ \\
Lysozyme & $0.6-0.7$ & 0.99
\end{tabular}

Taken from Chen et al. (2007) reviewed elsewhere (Scott et al. 1998), the simulated molecule experiences a potential that biases the trajectory to be consistent with the time-averaged experimental observable (Fig. 4d); its key variable is the averaging time. Recent examples of its use include analysis of information about structural heterogeneity encoded in NMR data, for example NOEs and ${ }^{3} \mathrm{~J}$ couplings (Tonelli et al. 2003; Allison and van Gunsteren 2009; Missimer et al. 2010), and RDCs (Hess and Scheek 2003). Tonelli et al. (2003), for example, used restrained time-averaged MD to determine an ensemble of structures for an RNA/ DNA hybrid duplex which enabled them to characterise the helix flexibility required for the binding of the duplex to ribonuclease $\mathrm{A}$.

An alternative to time-averaging is restrained ensembleaveraged MD, in which multiple configurations are simulated in parallel using a potential that biases the ensemble-averaged NMR parameters to agree with the experimental observable at each step of the simulation (Fig. 4e). The characterisation of structural heterogeneity from ensemble restrained MD was initially hampered by the inability of NOE to correctly identify the correct distribution of inter-proton distances (Bonvin and Brunger 1996) because of the non-linear averaging of this NMR parameter. This problem has now been overcome to a significant extent by using more appropriate NMR data, enabling this approach to generate quite realistic representations of the structural heterogeneity of proteins and nucleic acids (Clore and Schwieters 2004a; Lindorff-Larsen et al. 2005a; Richter et al. 2007). Initially these methods used $S^{2}$ and NOE data, however observables such as scalar couplings (Lindorff-Larsen et al. 2005b), trans hydrogen bond scalar couplings (Gsponer et al. 2006), and RDCs (Clore and Schwieters 2004a; De Simone et al. 2009; Fenwick et al. 2010, 2011) can also be used.

Ensemble restrained MD has been recently used to determine the structural heterogeneity of proteins of nucleic acids on the picosecond to millisecond timescale by exploiting the information contained in RDCs. Work by Lange et al. (2008) has shown that the motion in proteins on this timescale is mainly observed in loops, although slow motion is thought to be present in the backbone of ubiquitin (Lange et al. 2008). In a different but closely related study, a large amount of motion of the loop of the E2 enzyme Ube $2 \mathrm{~g} 2$ was observed, suggesting that the E2 enzyme may be regulated by the motion of this loop ( Ju et al. 2010). A conformational ensemble of GB3 and an ensemble produced by us have both shown that the motion of residues can be correlated (see below). For the B3 IgG binding domain of streptococcal protein $G$ it was shown that the fluctuations of the backbone dihedral of neighbouring residues are correlated (Clore and Schwieters 2004a; Bouvignies et al. 2005). As we will show, we have 
used a conceptually similar approach to reveal the presence of weak but statistically significant long-range correlated motion in an ensemble for the protein ubiquitin (Fenwick et al. 2011). Schwieters and Clore used ensemble simulations restrained by RDCs to study the motion of a Dickerson DNA dodecamer and showed that DNA is a relatively rigid molecule in terms of its overall macroscopic persistence length. They found, however, that the motion of the bases was of larger amplitude than that of the phosphate backbone. In Fig. 5, we show representations of Dickerson DNA from restrained MD $(N=1)$ and ensemble restrained $\operatorname{MD}(N=4)$. It can be seen that the motion of the DNA is more complex than simple fluctuations around the mean structure. These observations are consistent with the hypothesis that the flexibility of DNA has a functional role, enabling it to recognise a wide range of binding proteins (Schwieters and Clore 2007).

The function of biomolecules is intimately linked to molecular recognition and, as a result, the purpose of much current research is to describe kinetically and structurally the interactions of biomolecules. As ensemble approaches encode information that is not present in a single structural snapshot (Chaudhury and Gray 2008) they offer clear advantages over conventional methods of structure determination.

\section{Biomolecular recognition}

The study of molecular recognition is important not only to gain an appreciation of the underlying mechanisms of essential biological function, but also to aid the development and generation of new drugs (Lane 2001;

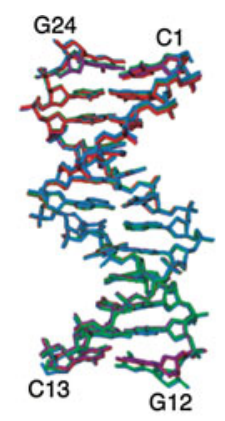

Four $\mathrm{N}=1$ ensembles

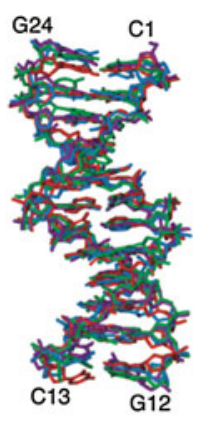

Ensemble $\mathrm{N}=4$

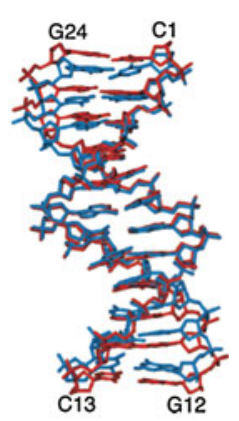

Average $\mathrm{N}=1$ Average $\mathrm{N}=4$
Fig. 5 Dickerson DNA restrained MD simulations $(N=1)$ and ensemble restrained MD simulations $(N=4)$. The four $N=1$ ensembles show that the average structure of the DNA is well defined by the experimental data, whereas the ensemble of $N=4$ shows that the data are consistent with motion to some extent. The average representations of the two ensembles, shown on the right, indicate that the two ensembles lead to noticeably different average structures. Taken from Schwieters and Clore (2007)
Aleksandrov and Simonson 2010). Four mechanistic models have been proposed for molecular recognition; these are the lock and key, induced fit, fluctuation fit, and conformational selection models. Recent reviews of the models themselves are already available in the literature (Ma et al. 1999; Kumar et al. 2000; Grunberg et al. 2004; Hammes et al. 2009; Zhou 2010; Vertessy and Orosz 2011). Here we will describe how conformational ensembles can be used to determine the extent to which the different models are appropriate for understanding the mechanism of molecular recognition for a given system.

The first of the models was proposed under the assumption that molecules were mainly static. In the model proposed by Fisher-the lock and key model-two molecules fit together because of their complementary shapes (Fischer 1894). The second model-the inducted fit model - proposed instead that one or both of the molecules changed conformation concomitantly with formation of the complex (Koshland 1958). These definitions distinguish the two possible mechanisms of binding for static molecules but they are not immediately applicable to most biomolecules because, as discussed above, these are appreciably dynamic.

Straub and Szabolcsi (1964) recast the lock and key model to accommodate the dynamic properties of biomolecules. In their new model, the fluctuation fit model, dynamic molecules pass through different conformational states and form a complex in a lock and key fashion when two complementary configurations occur (Straub and Szabolcsi 1964; Vertessy and Orosz 2011).

The induced fit mechanism can be rendered consistent with the presence of dynamics by refining its definition in order to distinguish it from fluctuation fit. Induced fit, when motion is present, implies that the formation of the complex generates a new configuration, one that is not sampled in the free state, for one or both molecules. Recently the fluctuation fit model has also been referred to as conformational selection. The idea of conformational selection, and that of population shift, were first proposed by the Nussinov group in a series of papers and this concept is now considered to be the leading model for molecular recognition (Ma et al. 1999; Tsai et al. 1999a, b; Kumar et al. 2000). We consider fluctuation fit and conformational selection to be equivalent and refer below to them both as conformational selection. In Fig. 6, we show how the two binding models are distinguished.

A unified model for molecular recognition has been proposed in which recognition proceeds via a three-step process (Grunberg et al. 2004), though in some cases some of the steps can be negligible. The first step is diffusion, followed by conformational selection, and the last step is induced fit. The authors argue that this mechanism fits the energetics and kinetics of complex 


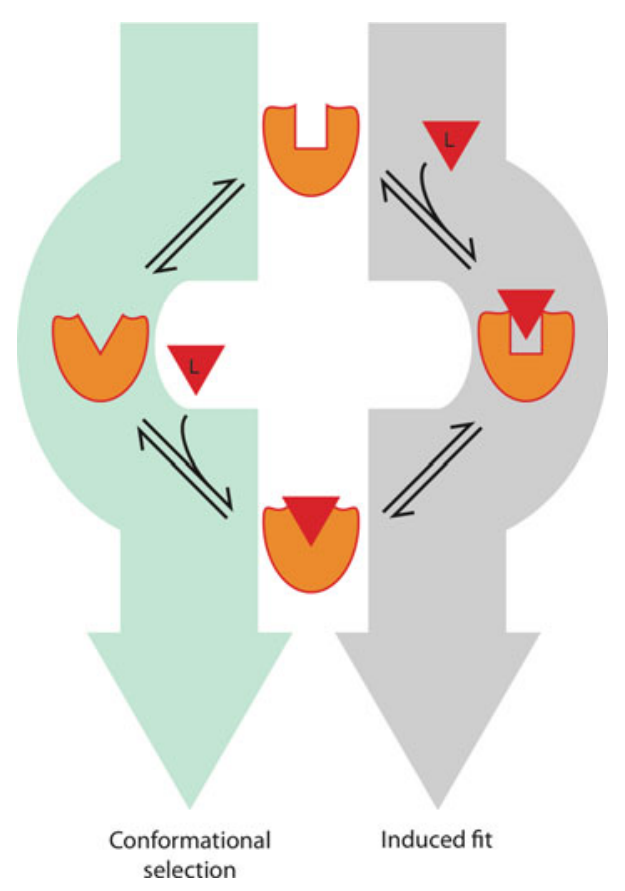

Fig. 6 Conformational selection and induced fit models of binding. The two different models are distinguished by the conformation of the binding site when the ligand binds. During conformational selection the ligand binds to the bound configuration of the binding site whereas during induced fit the bound form of the complex is formed after binding of the ligand

formation better than conformational selection or induced fit alone. Recently the relative weights of conformational selection and induced fit were analysed for ubiquitin binding (Wlodarski and Zagrovic 2009; Long and Brüschweiler 2011a); in agreement with this unified model of molecular recognition both conformational selection and induced fit were observed to be involved in the binding mechanism.

In the literature, the induced fit model is more prevalent than the conformational selection mechanism, because of the common observation that the crystal structure of the bound protein is different to that of the free molecule. Others have noted, and we stress here again, that a conclusion of induced fit because of different snapshots of the free and bound proteins is not warranted (Boehr et al. 2009). Similarly, the inability to detect low populated states does not mean that they do not exist. Recent advances enable the detection and characterisation of nearinvisible, low populated stable states in the native ensemble (Korzhnev et al. 2010). It will be seen below that structural heterogeneity can sometimes account for the observed differences without the need to invoke an induced fit mechanism. Discrimination of recognition mechanisms purely on the basis of static structures is not possible and the use of methods that provide information on structural heterogeneity can indeed be of great use.
Various mechanisms have been proposed to describe the binding behaviour of the three-domain protein AdK. In this protein, the two side domains need to close over the central domain for catalysis to occur. Early X-ray structures indicated that in the free form the molecule exists in an open state whereas binding to substrates or substrate analogues results in a closed state (Pai et al. 1977). This original report described the binding as induced fit but in a later NMR study it was observed that the side domains of AdK undergo substantial microsecond to millisecond conformational exchange in the absence of substrate (Shapiro et al. 2002) and that the substrate-bound form of the protein is compact. However, these results neither prove nor disprove the hypothesis that free AdK visits the closed state with any appreciable frequency. To determine whether conformational selection or induced fit best represent the mechanism by which AdK binds its substrates, a conformational ensemble is potentially a very powerful tool. If induced fit is the dominant mechanism for this protein the conformational ensemble would not contain closed configurations; if, instead, closed configurations can be found in the ensemble of the free protein it is then possible that AdK assumes the bound configuration by conformational selection.

Arora and Brooks (2007) performed MD simulations of AdK and observed that the protein does, indeed, assume the closed state, with only minor structural rearrangement required to obtain the bound configuration. Moreover, experimental evidence for AdK visiting the closed state in the absence of substrate comes from three configurations of a thermophilic AdK that were crystallised and are intermediate structural snapshots between the open and closed states (Henzler-Wildman et al. 2007). It would therefore seem that the dominant mechanism whereby AdK reaches the bound state, in terms of gross structural rearrangement, is conformational selection. It is clear from this example of AdK that conformational ensembles of the free state provide a simple way to determine which mechanism is dominant for a given system. Despite the clarity of the models above, the number of cases in which a given mechanism has been clearly demonstrated is limited. In the next section, we highlight some representative examples and discuss how they can be interpreted.

\section{Biomolecular recognition from ensembles}

The first examples of conformational selection mechanisms are starting to appear in the literature. MD simulations were used by Salsas-Escat and Stultz (2010) to generate ensembles of type III collagen that describe its conformational heterogeneity. They observed that collagen could adopt in the free state the configuration that it adopts in the 
active site of proteolytic enzyme CMMP8 that cleaves disordered peptides. Collagen contains additional cleavage sites for the proteolytic enzyme CMMP8 but these sites do not sample the bound configuration. These experiments suggest that proteolytic degradation of collagen is controlled by the formation of the correct collagen conformation, and the results are consistent with the experimentally observed cleavage pattern (Fields 1991).

Conformational selection has also been shown to be a viable molecular recognition model for nucleic acids. TAR RNA, for example, selects its ligands by conformational selection (Zhang et al. 2006). This RNA contains a bulge, which is a source of structural heterogeneity in the molecule. The motion of the bulge was analysed by use of RDCs with the help of MD simulations to generate a conformational ensemble that captures the dynamic sub-states of the system. Analysis of the ensemble revealed that the internal motion of TAR RNA was equivalent to the structural changes observed in X-ray structures of TAR RNA in complexes with its ligands.

Examples of induced fit are less abundant, although a disorder to order transition upon binding provides a good example of this mechanism. An MD approach was used to study the RNA binding protein TIS11d. This protein folds upon binding to its cognate RNA to regulate RNA degradation (Qin et al. 2009) as shown by NMR experiments (Hudson et al. 2004). Using MD simulations to study the unfolded state the authors observed that the distribution of configurations assumed by the bound state did not overlap with that corresponding to the unbound state. This example, where the induced fit mechanism seems to dominate, does not exclude the presence of the conformational selection mechanism, which could be also contribute to binding. As shown in Fig. 7, to be able to conclude which mechanism dominates for a particular binding reaction is key to determining the population of the bound conformation in the unbound state by using MD or ensemble MD restrained with appropriate NMR data.

A combination of conformational selection and induced fit has been observed in ubiquitin binding. More than 40 structures of ubiquitin in complex with binding partners are available and show that ubiquitin can adopt different configurations upon binding. Lange et al. (2008) determined a conformational ensemble of unbound ubiquitin from RDC and NOE data and were able to identify an ensemble member that was within $\sim 0.8 \AA$ of each of the bound structures of ubiquitin. This result showed that sampling the free state was sufficient to reach the configuration of the bound state in a conformational selection mechanism, with only minor induced fit changes to the backbone and side chain rotameric states. Wlodarski and Zagrovic (2009) further studied ubiquitin using global multidimensional scaling analysis to determine the relative weights of
Conformational selection

ubiquitin:hr1 complex

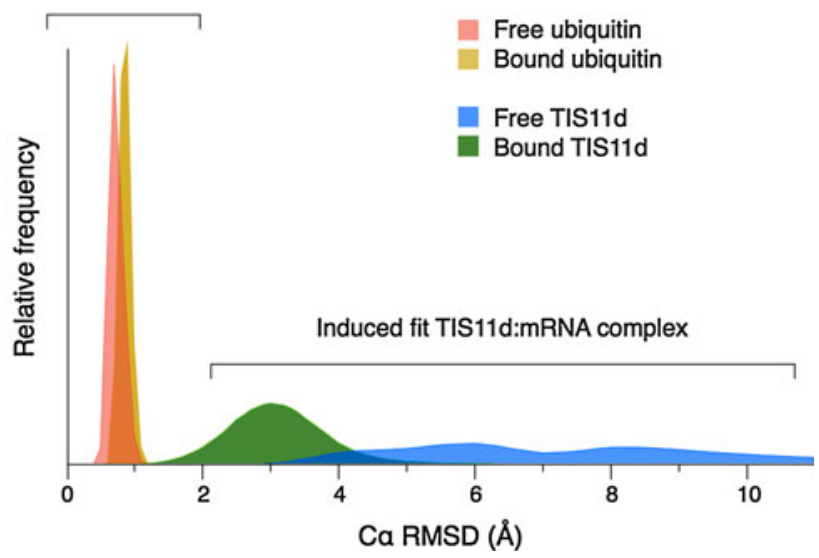

Fig. 7 Conformational selection and induced fit ensembles. Conformational selection for ubiquitin where the bound and unbound conformations share large overlap (yellow and red) and induced fit for TIS11d where there is very little overlap of the distributions (green and blue). Data for ubiquitin adapted from Qin et al. (2009). Ubiquitin data taken from Fenwick et al. (2011) and MoDEL (Meyer et al. 2010). The distributions of pairwise RMSD to the average bound structure are scaled to have the same volume

induced fit and conformational sampling, and concluded that the role of induced fit was only marginally lower than that of conformational selection. We recently generated a new conformational ensemble of ubiquitin using RDCs and NOEs and identified ensemble members that are extremely similar to the bound states of ubiquitin $(\sim 0.5 \AA)$; this result suggests that conformational selection is likely to play a larger role than induced fit in the molecular recognition of ubiquitin (Fenwick et al. 2011). Recently Long and Brüschweiler have used a reweighting method to study the interplay between ubiquitin and UIM during complex formation. Their innovate technique gives further evidence of the role of conformational selection in the binding of ubiquitin partners via a population redistribution i.e. population shift mechanism (Long and Brüschweiler 2011a). We highlight the differences between this example of conformational selection with that of induced fit for TIS11d in Fig. 7. It can be seen that in the case of conformational selection the bound and free states have low and similar RMSD profiles, whereas for the induced fit case sampling of the bound and free states of TIS11d is quite different.

Gaspari et al. (2010) have shown that the canonical serine protease inhibitor lock and key model can be explained by conformational selection. They generated conformational ensembles from $S^{2}$ and NOE data for two peptide proteases inhibitors (SGCI and SGTI) and found in both cases that the conformational ensemble of the free states contained configurations corresponding to the structures of the inhibitors bound to the proteases (Gaspari et al. 2010). 
For RNA-protein interactions, it has been proposed that induced fit makes the largest contribution to molecular recognition because in many cases the solution structure of the RNA is markedly different from that observed in the complexes with RNA-binding proteins (Williamson 2000). Despite this, counter examples can be found for which the dominant mechanism seems to be lock and key. Wright and co-workers show that a lock and key mechanism can explain the binding of finger 4 of the transcription factor IIIA to 5S RNA. A second report of lock and key for RNA has also become available in which one of the molecules is static and does not change its conformation upon binding. In this case the lock and key hypothesis was based on the solution structure of 7SK-SL RNA free and in complex with argininamide (Dethoff and Al-Hashimi 2010; Durney and D'Souza 2010), which showed no evidence of reorganisation upon binding. Moreover, mutations that freeze out motion of the related TAR RNA have been shown to increase the binding affinity (Stelzer et al. 2010). These examples highlight the possibility that the static lock and key mechanism may apply in some cases, although examples are limited.

\section{Allostery and correlated motion}

In the above examples, we have ignored the role of allostery in molecular recognition. Allostery is the process by which the affinity of a binding site for a ligand is affected by the binding of a second ligand in a different, distant, site. This process requires the transfer of structural and/or dynamic information across the macromolecule through, potentially, correlated conformational changes. Simple contact models can characterize networks of this type for some biomolecules but others seem to operate via more complex coupling mechanisms (Daily et al. 2008) that can include changes in quaternary structure (Daily and Gray 2009).

The Nussinov group recently emphasised the importance of allostery in signal transduction and transcriptional control (Ma et al. 2010). In their opinion, all interactions can potentially have allosteric consequences, because of the nature of population shift, and can result in highly complex and redundant networks. Using these ideas, they were able to rationalise the complexity of biomolecular interaction networks that operate in transcriptional regulation (Pan et al. 2009). This work has recently been reviewed (Pan et al. 2010).

Three different idealised models for allostery-the MWC model, the KNF model, and the more recently suggested conformational spread model-have been proposed to explain binding allostery (Kumar et al. 2000; Liu et al. 2006; Okazaki and Takada 2008; Tsai et al. 2008, 2009; Whitley and Lee 2009). These models consider that only two well-defined conformations for each binding site, corresponding to the free and bound states, can be adopted. The MWC model assumes that these two conformations are in equilibrium and that the motion of each of the binding sites is fully correlated; it is conceptually related to the conformational selection mechanism of molecular recognition as the bound conformation at both binding sites is visited even in the absence of ligand. Gunasekaran et al. (2004) first proposed this idea, in which allostery proceeds via a population shift of the ensemble. The KNF model is instead conceptually related to the induced fit mechanism of molecular recognition; it does not require the presence of motion in the free state and suggests that ligand binding induces a change of structure in the first binding site that causes the formation of one or more well-defined intermediate states in which affinity for the second ligand is altered through the propagation of conformational changes across the structure of the protein. The conformational spread model and Eigen's scheme (Eigen 1967) can be regarded as the fully enumerated intermediate states between the MWC and KNF models. These schemes are shown in Fig. 8.

It is interesting to consider the implications of these models for the motion of allosteric biomolecules in the absence of their ligands. The MWC model, which explicitly considers the dynamics of the free state, invokes strong correlation of the motions at each binding site. The KNF model, instead, only requires binding in the first site to influence the affinity of the second binding site for its ligand. These observations indicate that careful analysis of the structural heterogeneity of the free state using conformational ensembles, together with knowledge of the structure of the bound state, potentially enables the mechanism by which binding allostery operates for specific systems to be determined. It can be seen from Fig. 9 that an accurate characterization of the structural heterogeneity of the free and bound states in terms of a conformational ensemble can provide information on the model that applies to a specific allosteric protein.

In the absence of experimentally validated conformational ensembles, double mutant cycles are one of the strongest experimental validations of allosteric channels and are routinely used in their investigation. These types of data can be useful in that they can determine if cooperative channels and mechanisms are present. In some cases they can indicate which residues are involved in such channels. Determination of the underlying mechanisms is more difficult, however, and requires atomic level descriptions of biomolecular motion. Istomin et al. (2008) have used this type of analysis and observed clear channels of communication between residues separated by non-sequential residues in many different proteins. In an NMR approach Mayer et al. (2003) combined NMR relaxation data for ten mutants of the B1 IgG binding domain of streptococcal 


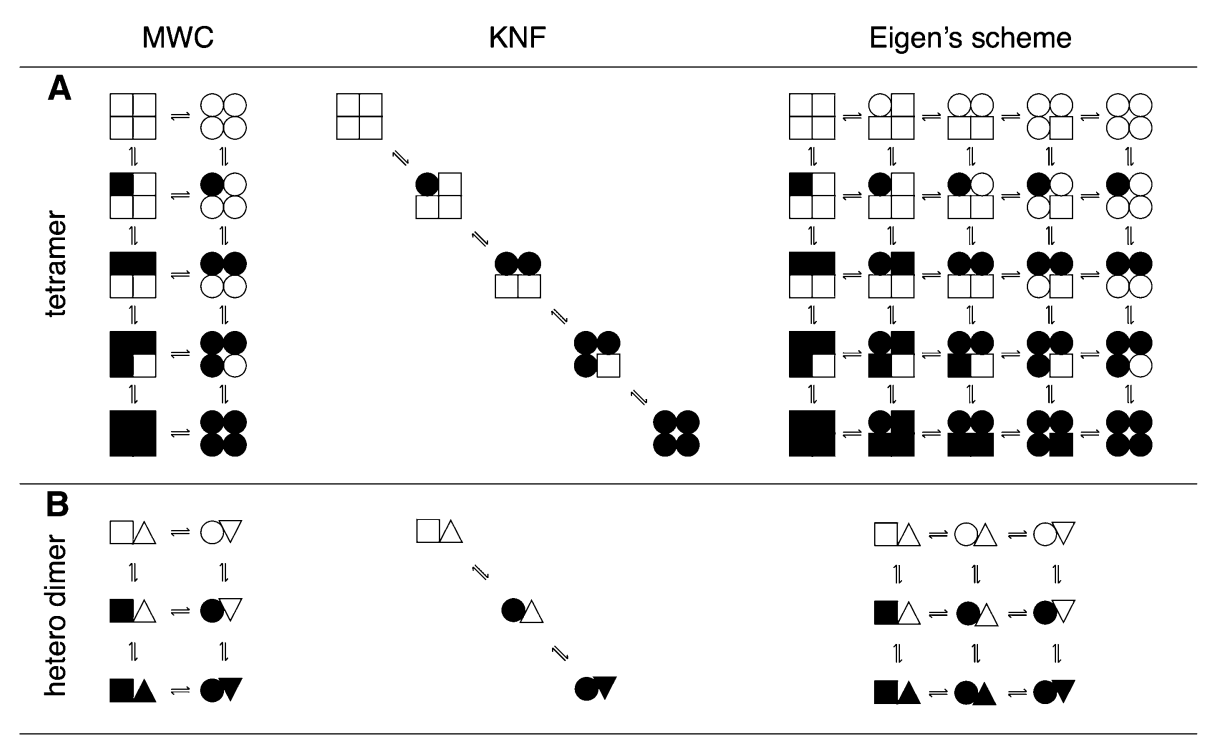

Fig. 8 Models of allostery. The schemes represent the identity of the conformations of a tetrameric (a) and heterodimer (b) allosteric protein that are present in solution as the concentration of ligand is increased from top to bottom. Ligand binding is represented as a change in color in the subunit from white to black whereas conformational change is represented by a change in shape; the populations of the various conformations are not represented. In the MWC model the species in solution do not change but their populations shift as a consequence of ligand binding; as only two

protein $\mathrm{G}$ and observed that the backbone order parameters varied less than would be expected if the residues fluctuated independently, suggesting widespread concerted motion of pairs of residues. This claim is controversial and could not be substantiated by MD simulations of this and other proteins (Lange et al. 2005).

\section{Biomolecular allostery from ensembles}

Descriptions of allostery from ensembles can be indicative of correlated motion. Because of the challenges in directly determining time-resolved coordinates from experiments, detection of correlations between the motion of residues distant in sequence has remained elusive. As described above, conformational ensembles are most often used for this purpose. They combine experimental (NMR) data with $\mathrm{MD}$, and enable protein motion to be characterized at atomic resolution.

Once the ensembles are generated, there are different ways of determining whether they contain correlated motion. One of the most important considerations is the frame of reference for extracting the motion. If Cartesian coordinates are chosen, the first difficulty is choosing the correct alignment frame for the ensemble members, because it has been shown that a poor choice of reference frame can lead to artefactual results (Theobald and Wuttke possible states are possible there is a strong correlation between the conformation of each subunit. In the KNF model, instead, ligand binding causes a local conformational change in the subunit, that influences the affinity of the other subunits for the ligand without the need to invoke structural changes; as the conformational changes in the various binding sites are not concerted this model requires a weaker correlation between the conformations of each subunit. The general scheme of Eigen, where a full permutation of the states is considered, is also shown (right)

2008). In Cartesian space, the standard analysis is computation of the distance correlation matrix; although this method is sensitive to large displacements, it does not report on the nature of the hinges that give rise to the observed displacements. Thus, if one is interested in obtaining mechanistic information on the channels of communication an alternative coordinate system is more appropriate. The choice of internal coordinates (dihedral space) is natural for proteins because most of their motion is due to fluctuations in dihedral angles. Correlated motion can be analysed in dihedral space and can be used to identify hinge regions. Thorough analysis of correlated motion requires analysis in both reference frames, although it is preferable to use internal coordinates when trying to understand mechanistic details and to elucidate allosteric channels (Clore and Schwieters 2004a; Showalter and Brüschweiler 2007; Li et al. 2009). We compare these two methods in Fig. 10 for an ensemble of ubiquitin that contains both short and long-range correlated motion.

Analysis of MD simulations has become commonplace in Cartesian space, with examples covering a large range of proteins. Dihydrofolate reductase has non-additive behaviour in double mutant cycles and MD simulations (Agarwal et al. 2002; Rajagopalan et al. 2002), in which the affect of mutations can be rationalised in terms of the small structural changes and specific rearrangements of the hydrogen bonds (Rod et al. 2003). Antibody conformations in the 


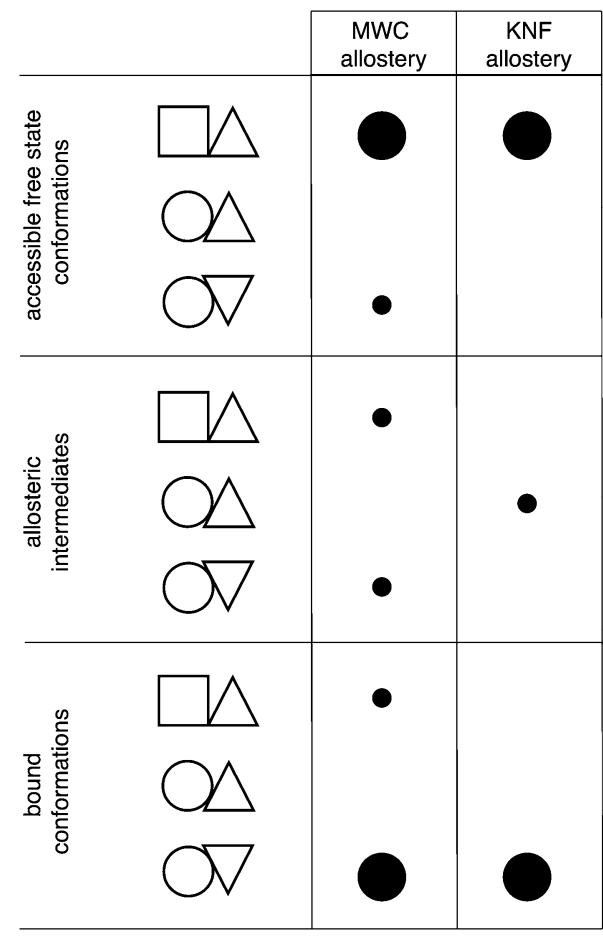

Fig. 9 The scheme represents the conformational states that are populated to a non-negligible extent in the free state, in the intermediate state proposed by the KNF model and in the bound state. It can be seen that an accurate characterization of the structural heterogeneity of the free and bound states in terms of a conformational ensemble can provide information on the model that applies to a specific allosteric protein. The size of the symbols indicates which are the major and minor states

free and bound forms are also significantly different. MD simulations of the unbound antibody were analysed to determine which motion is correlated between the various regions. It was observed that intra-domain correlations exist on timescales of $40 \mathrm{ps}$ and above, and that interdomain correlations between the heavy and light chains were not present below 60 ps (Viswanathan et al. 2000). These results suggest that allostery exists between the heavy and light chains of the antibody and may be important in communicating the bound signal through the antibody to the Fc region, which leads to the correct immune response.

Recently an MD study on the ferredoxin protein motif has revealed that mutations in a loop region distant ( $\sim 20 \AA$ ) from the active site have an appreciable affect on the redox potential at the iron-sulphur cluster-binding region (Nechushtai et al. 2011). The loop truncation was observed to have limited effect on the structure of the molecule as determined by X-ray crystallographic studies; the authors propose that changes in motion, propagated via a channel, are responsible for the change in redox potential. MD simulations were used to characterise the motion of the system and the ensembles generated were analysed to uncover correlated motion. The simulations predicted the propagation of motion between strands of the $\beta$-sheet, consistent with the $\beta$-lever motion (Fenwick et al. 2011) that has been observed to couple motion across $\beta$-strands.

The drug Hoechst 33258 binds to duplex DNA. NMR experiments could not detect the presence of singly bound Hoechst 33258 to DNA and, instead, showed that only the doubly bound Hoechst 33258 to DNA species seems to exist in solution (Searle and Embrey 1990). Extended MD simulations of the 1:1 and 2:1 complex of the drug and DNA showed that binding allostery is primarily a consequence of the transfer of dynamic information rather than of structural change (Harris et al. 2001). A similar example comes from the ensembles of TAR-RNA determined by the Al-Hashimi group (Zhang et al. 2007). Careful experimental investigations combined with modelling enabled the observation of correlated motion that biases transitions along predetermined conformational pathways (Fig. 11). In this case the pathways of motion could be directly determined from the experimental data, and by selecting frames from ensembles determined using unbiased MD it was possible to visualise correlated motion. It seems that the bulge in TAR-RNA is responsible for the pathways of motion, because the same pathway bias was not observed for RNA without the bulge. Furthermore, it was possible to place the X-ray structures of the RNA on the pathways showing that they link biologically relevant states.

Illustration of correlations in dihedral space has primarily been applied to understanding of the local motion in the backbones of proteins. Indeed the first MD simulations reported for BPTI showed backbone correlations from analysis of the trajectory in dihedral space (McCammon et al. 1977). These correlations were observed to minimise the structural displacements that would otherwise be caused by large-amplitude bond rotation in the backbone. The crankshaft motion is caused by the rigidity of the peptide plane and couples $\psi_{i-1}$ with $\phi_{i}$. The authors also observed that the crankshaft motion also operates between the $\chi$ angles of aromatic residues to enable motion of the side chain without flipping of the aromatic side chain (McCammon et al. 1977). These correlations have also been described by use of modern very long MD simulations with state of the art force fields (Fitzgerald et al. 2007; Li et al. 2009).

Ensembles generated with experimental data are also known to reproduce short and medium-range correlated motion. Clore and Schwieters (2004a) were able to obtain direct evidence of the existence of crankshaft motion from ensemble MD simulations of the B3 IgG binding domain of streptococcal protein $\mathrm{G}$ restrained by RDCs. A second study showed that a description of the dynamics of the same protein using the 3D Gaussian axial fluctuation model, that was fit to RDCs, gave better agreement with experimental 

dihedral and Cartesian space for the ERNST ensemble (Fenwick et al. 2011). Top left, the structure of ubiquitin, indicating the organisation of the $\beta$-strands and the degree of long-range structural correlation (red indicates high correlation, green $\mathrm{C} \alpha$ distance matrix of ubiquitin close in space. Results are shown from correlation analysis for a conformational ensemble of ubiquitin in Cartesian space (bottom left) and in dihedral range correlations are indicated with green dashed lines, and long-range correlations with red dashed circles
Fig. 10 Correlations in no correlation). Top right, the indicates which residues are space (bottom right). Short-
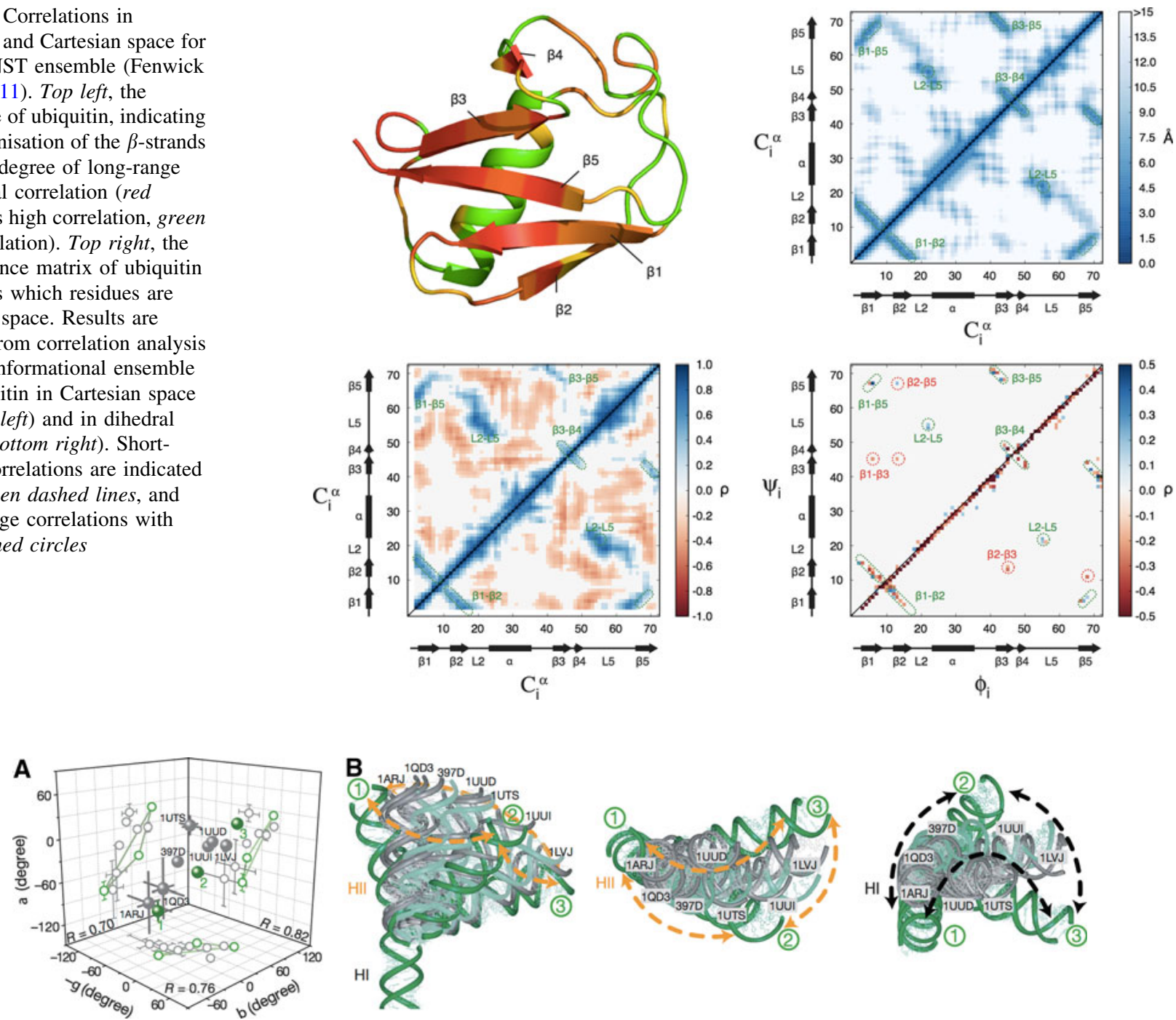

Fig. 11 TAR RNA biased transitions. a The three TAR dynamic conformers (green) and the TAR conformation (grey) bound to peptide derivatives of Tat and different small molecules. Shown on each 2D plane is the correlation coefficient $(R)$ between angles for the ligand-bound conformations. b Comparison of the three TAR dynamic conformers (green) and ligand-bound TAR conformations

NMR results when correlated motion between residues in opposite strands was invoked (Bouvignies et al. 2005). This study provided evidence that hydrogen bonds transfer motion between strands, which presumably could also operate to couple motion up and down helices.

Work in our own laboratory has led to a detailed mechanistic description of the pathway that connects two loops involved in molecular recognition of ubiquitin, as shown in Fig. 12. Using the restrained MD approach with the large amount of experimental data collected for ubiquitin (Lakomek et al. 2006; Lange et al. 2008), we have been able to identify weak long-range correlated motion in ubiquitin that conserves the local structure and enables the propagation of conformational change across the structure (grey). Sub-conformers along the linear pathway linking conformers are shown in light green, and the direction of the trajectory is shown with arrows. Left panel, horizontal view after superposition of HI; middle and right panels, vertical view down and up the helix axis of $\mathrm{HI}$ and HII after superposition of $\mathrm{HI}$ and HII, respectively. Taken from Zhang et al. (2007)

of the protein. These observations can be rationalised in terms of the local peptide geometry interacting with the hydrogen-bonding network. Moreover, motion in the $\beta$-strands is manifest in a specific sequence of structural changes that lead to the weak coupling of the motion of the two binding loops in ubiquitin.

Our analysis of the conformational ensemble of ubiquitin revealed that correlated backbone motion both conserves the structure of ubiquitin and provides a pathway for transfer of structural and dynamic information. The pathway is very detailed in the sense that the combination of experimental data with the MD force field gives rise to a pathway of dihedral rotations that can be connected in a linear sequence of events (Fenwick et al. 2011). 

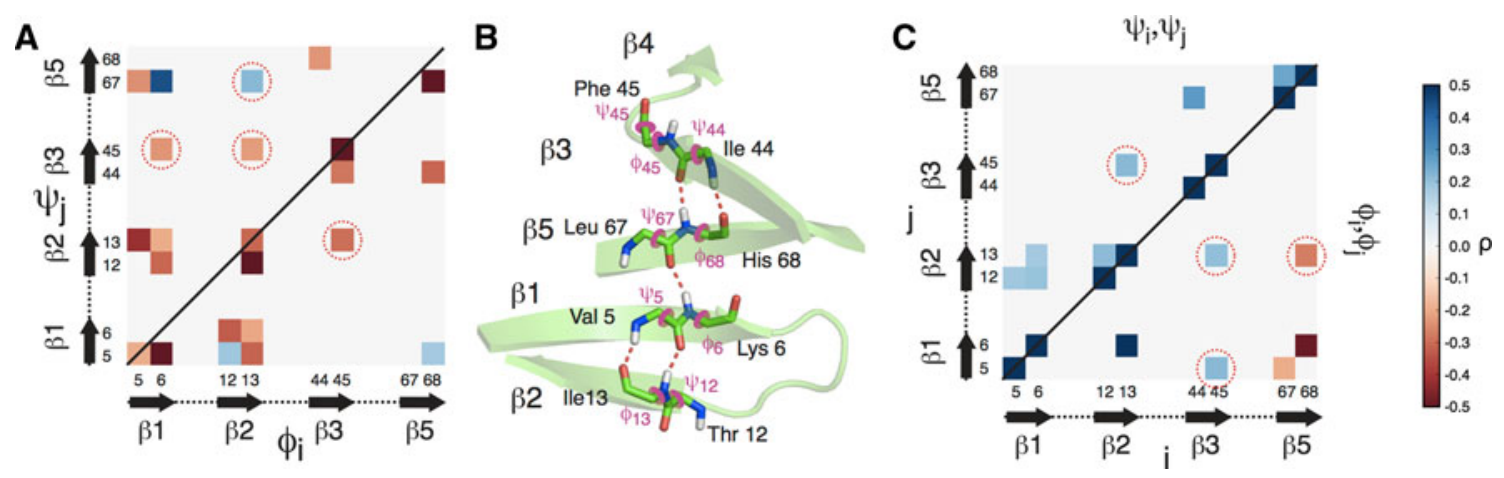

Fig. 12 Long-range correlations in a conformational ensemble of ubiquitin that create a channel between the two loops involved in molecular recognition. a Circular correlation coefficients $(\rho)$ of $\varphi$ and $\psi$ of residues that are part of the surface patch of ubiquitin involved in binding to ubiquitin binding domains. b Representation of the

\section{Challenges in characterizing molecular recognition and allostery}

In the preceding sections, we have detailed the use of ensembles to elucidate mechanisms of binding and how the use of ensembles can help to identify the model of molecular recognition. We have also presented the current view of allostery with relevant examples to show that ensembles can play a pivotal role in dissecting the mechanisms that govern signal transduction. The use of the ensembles is not limited to globular folded proteins and nucleic acids but also finds applications in disordered and highly flexible biomolecules. Despite recent theoretical developments, limitations in ensemble generation still limit the resolution and quality of ensembles for these later systems. The current limit is not with the methods of analysis, but rather relate to the difficulty in determining ensemble representations.

One limitation is the sampling problem. In the applications above, which used unrestrained MD simulations to generate the ensembles, the timescale of the simulation required is generally 10 times longer than the process of interest that one is trying to study. For small globular proteins, with high-frequency motion only, MD simulations are now able to capture the required motion (Showalter and Brüschweiler 2007; Li and Brüschweiler 2009; Lange et al. 2010). However, for large or extended molecules, i.e. molecules with multiple domains, for which large amplitude motion is possible, conventional MD does not sample enough space in the practical timescales of these simulations. Advanced sampling techniques are now being used to reduce this limitation and have been shown to improve the agreement between simulations and experimental data (Lange et al. 2006; Allison and van Gunsteren 2009; Markwick et al. 2009). Ensemble restrained methods do not suffer from this problem as much as unrestrained $\mathrm{MD}$, because simulated annealing is often used to explore corresponding $\beta$-strands showing the dihedral angles that sense the channel. c Correlation between $\varphi_{i}$ and $\psi_{j}$ of residues that are part of the network. Long-range correlations involving distant residues are indicated by red dashed circles (a, c). Taken from Fenwick et al. (2011)

conformational space, and the experimental restraints can be seen as generating a system-specific force field.

NMR data are not the only data that can be used to generate ensembles for study of molecular recognition. Some recent ensembles have been generated by use of small-angle X-ray scattering (SAXS) (Bernado et al. 2010). Other experimental techniques are not so limited by size, or improve in resolution as size increases. Thus, the use of FRET distance distributions, SAXS, small-angle neutron scattering (SANS), and other techniques may be of greater benefit than NMR for these systems. Indeed SAXS and NMR seem to give a consistent view for systems even as challenging as unfolded proteins (Bernado and Blackledge 2009). These methods tend to be used when the sizes of the complexes are too large to be studied by NMR. NMR is still favoured in many cases because of the detailed site-specific information that can be extracted by use of this technique.

Theoretical interpretation of the data can still be a problem. One example that comes from our laboratory is the use of RDCs in the presence of flexibility. We have shown that conventional methods for determining motion from RDCs cannot be used to interpret the motion of multidomain biomolecules. Until recently, only simple models were available for domain motion (Ryabov and Fushman 2006). Current research is providing solutions to this problem and will enable the motion of these systems to be characterised at atomic resolution (Esteban-Martin et al. 2010; Huang and Grzesiek 2010).

The generation of ensembles for unfolded states is very difficult. Statistical coil models, in which ensembles were generated by randomly selecting dihedrals from loop libraries, had some success (Bernado et al. 2005; Jha et al. 2005; Bernado and Blackledge 2009). Despite the local properties being approximately correct for these ensembles, there is room for improvement (Nodet et al. 2009). Refining these ensembles with RDCs enabled the local refinement of these 
structures for ubiquitin (Esteban-Martin et al. 2010) whereas some corrections for longer-range contacts were still needed to generate ensembles of $\alpha$-synuclein, although progress is being made (Bernado et al. 2005; Salmon et al. 2010).

Two remaining challenges are to accurately determine the population weights of the ensemble members and the timescale of their interconversion. It is very challenging to determine relevant population weights with certainty from experimental data but possibility of generating a free energy landscape from distances measured using FRET was recently demonstrated (Schuetz et al. 2010). Advances of this type together with improvements in ensemble methods will lead to determination of ensembles of larger or elaborate biomolecules.

\section{Perspective}

How do biomolecules recognise their partners and lead to biological responses? We have in this review provided an account of how conformational ensembles determined by combining NMR data with molecular simulations can be used to determine the mechanism by which molecular recognition and its consequences occur in biological macromolecules. As we imply above, the field is still in its infancy and there are many types of structural heterogeneity in macromolecules that are challenging to experimentally characterize, because of difficulties in interpretation of the experimental data or because of the absence of algorithms for efficient generation of conformational ensembles at high resolution. There is no doubt, however, that as better experimental and computational methods become available the combination of experimental data with molecular simulations will enable us to better understand the mechanism of molecular recognition and binding allostery and to exploit this new knowledge for discovery of better and less expensive drugs by structure and dynamics-based drug design.

Open Access This article is distributed under the terms of the Creative Commons Attribution Noncommercial License which permits any noncommercial use, distribution, and reproduction in any medium, provided the original author(s) and source are credited.

Note added in proof During the proof stage of the review the Brüschweiler group published a description of allostery for BPTI, adding another important example of how weak correlations propagate signals in remote regions of proteins (Long and Brüschweiler 2011b).

\section{References}

Agarwal PK, Billeter SR, Rajagopalan PT, Benkovic SJ, HammesSchiffer S (2002) Network of coupled promoting motions in enzyme catalysis. Proc Natl Acad Sci USA 99:2794-2799
Aleksandrov A, Simonson T (2010) Molecular dynamics simulations show that conformational selection governs the binding preferences of imatinib for several tyrosine kinases. J Biol Chem 285:13807-13815

Allerhand A, Doddrell D, Glushko V, Cochran DW, Wenkert E, Lawson PJ, Gurd FR (1971) Conformation and segmental motion of native and denatured ribonuclease $\mathrm{A}$ in solution. Application of natural-abundance carbon-13 partially relaxed Fourier transform nuclear magnetic resonance. J Am Chem Soc 93:544-546

Allison JR, van Gunsteren WF (2009) A method to explore protein side chain conformational variability using experimental data. Chemphyschem 10:3213-3228

Arora K, Brooks CL (2007) Large-scale allosteric conformational transitions of adenylate kinase appear to involve a populationshift mechanism. Proc Natl Acad Sci USA 104:18496-18501

Bahar I, Lezon TR, Yang LW, Eyal E (2010) Global dynamics of proteins: bridging between structure and function. Annu Rev Biophys 39:23-42

Beckstein O, Denning EJ, Perilla JR, Woolf TB (2009) Zipping and unzipping of adenylate kinase: atomistic insights into the ensemble of open $\leftrightarrow$ closed transitions. J Mol Biol 394:160-176

Bernado P, Blackledge M (2009) A self-consistent description of the conformational behavior of chemically denatured proteins from NMR and small angle scattering. Biophys J 97:2839-2845

Bernado P, Bertoncini CW, Griesinger C, Zweckstetter M, Blackledge $M$ (2005) Defining long-range order and local disorder in native alpha-synuclein using residual dipolar couplings. J Am Chem Soc 127:17968-17969

Bernado P, Modig K, Grela P, Svergun DI, Tchorzewski M, Pons M, Akke M (2010) Structure and dynamics of ribosomal protein L12: an ensemble model based on SAXS and NMR relaxation. Biophys J 98:2374-2382

Best RB, Vendruscolo M (2004) Determination of protein structures consistent with NMR order parameters. J Am Chem Soc 126:8090-8091

Blagoev KB, Alexandrov BS, Goodwin EH, Bishop AR (2006) Ultra-violet light induced changes in DNA dynamics may enhance TT-dimer recognition. DNA Repair (Amst) 5:863-867

Boehr DD, Nussinov R, Wright PE (2009) The role of dynamic conformational ensembles in biomolecular recognition. Nat Chem Biol 5:789-796

Bonvin AM, Brunger AT (1996) Do NOE distances contain enough information to assess the relative populations of multi-conformer structures? J Biomol NMR 7:72-76

Bonvin AM, Boelens R, Kaptein R (1994) Time- and ensembleaveraged direct NOE restraints. J Biomol NMR 4:143-149

Bouvignies G, Bernado P, Meier S, Cho K, Grzesiek S, Brüschweiler $\mathrm{R}$, Blackledge M (2005) Identification of slow correlated motions in proteins using residual dipolar and hydrogen-bond scalar couplings. Proc Natl Acad Sci USA 102:13885-13890

Chaudhury S, Gray JJ (2008) Conformer selection and induced fit in flexible backbone protein-protein docking using computational and NMR ensembles. J Mol Biol 381:1068-1087

Chen Y, Campbell SL, Dokholyan NV (2007) Deciphering protein dynamics from NMR data using explicit structure sampling and selection. Biophys J 93:2300-2306

Chu JW, Voth GA (2005) Allostery of actin filaments: molecular dynamics simulations and coarse-grained analysis. Proc Natl Acad Sci USA 102:13111-13116

Clore GM, Schwieters CD (2004a) Amplitudes of protein backbone dynamics and correlated motions in a small alpha/beta protein: correspondence of dipolar coupling and heteronuclear relaxation measurements. Biochemistry 43:10678-10691

Clore GM, Schwieters CD (2004b) How much backbone motion in ubiquitin is required to account for dipolar coupling data 
measured in multiple alignment media as assessed by independent cross-validation? J Am Chem Soc 126:2923-2938

Clore GM, Schwieters CD (2006) Concordance of residual dipolar couplings, backbone order parameters and crystallographic B-factors for a small alpha/beta protein: a unified picture of high probability, fast atomic motions in proteins. J Mol Biol 355:879-886

Daily MD, Gray JJ (2009) Allosteric communication occurs via networks of tertiary and quaternary motions in proteins. PLoS Comput Biol 5:e1000293

Daily MD, Upadhyaya TJ, Gray JJ (2008) Contact rearrangements form coupled networks from local motions in allosteric proteins. Proteins 71:455-466

de Groot BL, Hayward S, van Aalten DM, Amadei A, Berendsen HJ (1998) Domain motions in bacteriophage T4 lysozyme: a comparison between molecular dynamics and crystallographic data. Proteins 31:116-127

De Simone A, Richter B, Salvatella X, Vendruscolo M (2009) Toward an accurate determination of free energy landscapes in solution states of proteins. J Am Chem Soc 131:3810-3811

Deniz AA, Dahan M, Grunwell JR, Ha T, Faulhaber AE, Chemla DS, Weiss S, Schultz PG (1999) Single-pair fluorescence resonance energy transfer on freely diffusing molecules: observation of Forster distance dependence and subpopulations. Proc Natl Acad Sci USA 96:3670-3675

Deniz AA, Laurence TA, Beligere GS, Dahan M, Martin AB, Chemla DS, Dawson PE, Schultz PG, Weiss S (2000) Single-molecule protein folding: diffusion fluorescence resonance energy transfer studies of the denaturation of chymotrypsin inhibitor 2. Proc Natl Acad Sci USA 97:5179-5184

Dethoff EA, Al-Hashimi HM (2010) A rare example of lock-and-key RNA recognition. J Mol Biol 404:553-554

Durney MA, D’Souza VM (2010) Preformed protein-binding motifs in 7SK snRNA: structural and thermodynamic comparisons with retroviral TAR. J Mol Biol 404:555-567

Eigen M (1967) Kinetics of reaction control and information transfer in enzymes and nucleic acids. Nobel Symp 5:333-369

Esteban-Martin S, Fenwick RB, Salvatella X (2010) Refinement of ensembles describing unstructured proteins using NMR residual dipolar couplings. J Am Chem Soc 132:4626-4632

Faure P, Micu A, Perahia D, Doucet J, Smith JC, Benoit JP (1994) Correlated intramolecular motions and diffuse X-ray scattering in lysozyme. Nat Struct Biol 1:124-128

Faustino I, Perez A, Orozco M (2010) Toward a consensus view of duplex RNA flexibility. Biophys J 99:1876-1885

Fenwick RB, Esteban-Martiín S, Salvatella X (2010) Influence of experimental uncertainties on the properties of ensembles derived from NMR residual dipolar couplings. J Phys Chem Lett 1:3438-3441

Fenwick RB, Esteban-Martin S, Richter B, Lee D, Walter KFA, Milovanovic D, Becker S, Lakomek NA, Griesinger C, Salvatella X (2011) Weak long-range correlated motions in a surface patch of ubiquitin involved in molecular recognition. J Am Chem Soc 133:10336-10339

Fields GB (1991) A model for interstitial collagen catabolism by mammalian collagenases*. J Theor Biol 153:585-602

Fischer E (1894) Einfluss der configuration auf die Wirkung der enzyme. Berichte der Deutschen Chemischen Gesellschaft 27:2985-2993

Fitzgerald JE, Jha AK, Sosnick TR, Freed KF (2007) Polypeptide motions are dominated by peptide group oscillations resulting from dihedral angle correlations between nearest neighbors. Biochemistry 46:669-682

Frank AT, Stelzer AC, Al-Hashimi HM, Andricioaei I (2009) Constructing RNA dynamical ensembles by combining MD and motionally decoupled NMR RDCs: new insights into RNA dynamics and adaptive ligand recognition. Nucleic Acids Res 37:3670-3679

Frauenfelder H, Mezei F (2010) Neutron scattering and protein dynamics. Acta Crystallogr D Biol Crystallogr 66:1229-1231

Frauenfelder H, Chen G, Berendzen J, Fenimore PW, Jansson H, McMahon BH, Stroe IR, Swenson J, Young RD (2009) A unified model of protein dynamics. Proc Natl Acad Sci USA 106:5129-5134

Gaspari Z, Varnai P, Szappanos B, Perczel A (2010) Reconciling the lock-and-key and dynamic views of canonical serine protease inhibitor action. FEBS Lett 584:203-206

Goto NK, Skrynnikov NR, Dahlquist FW, Kay LE (2001) What is the average conformation of bacteriophage T4 lysozyme in solution? A domain orientation study using dipolar couplings measured by solution NMR. J Mol Biol 308:745-764

Grunberg R, Leckner J, Nilges M (2004) Complementarity of structure ensembles in protein-protein binding. Structure 12:2125-2136

Gsponer J, Hopearuoho H, Cavalli A, Dobson CM, Vendruscolo M (2006) Geometry, energetics, and dynamics of hydrogen bonds in proteins: structural information derived from NMR scalar couplings. J Am Chem Soc 128:15127-15135

Gunasekaran K, Ma B, Nussinov R (2004) Is allostery an intrinsic property of all dynamic proteins? Proteins 57:433-443

Hammes GG, Chang YC, Oas TG (2009) Conformational selection or induced fit: a flux description of reaction mechanism. Proc Natl Acad Sci USA 106:13737-13741

Harris SA, Gavathiotis E, Searle MS, Orozco M, Laughton CA (2001) Cooperativity in drug-DNA recognition: a molecular dynamics study. J Am Chem Soc 123:12658-12663

Henzler-Wildman K, Kern D (2007) Dynamic personalities of proteins. Nature 450:964-972

Henzler-Wildman KA, Thai V, Lei M, Ott M, Wolf-Watz M, Fenn T, Pozharski E, Wilson MA, Petsko GA, Karplus M, Hubner CG, Kern D (2007) Intrinsic motions along an enzymatic reaction trajectory. Nature 450:838-844

Hess B, Scheek RM (2003) Orientation restraints in molecular dynamics simulations using time and ensemble averaging. J Magn Reson 164:19-27

Hodgkin AL, Keynes RD (1955) The potassium permeability of a giant nerve fibre. J Physiol 128:61-88

Huang JR, Grzesiek S (2010) Ensemble calculations of unstructured proteins constrained by RDC and PRE data: a case study of ureadenatured ubiquitin. J Am Chem Soc 132:694-705

Hub JS, de Groot BL (2009) Detection of functional modes in protein dynamics. PLoS Comput Biol 5:e1000480

Hudson BP, Martinez-Yamout MA, Dyson HJ, Wright PE (2004) Recognition of the mRNA AU-rich element by the zinc finger domain of TIS11d. Nat Struct Mol Biol 11:257-264

Istomin AY, Gromiha MM, Vorov OK, Jacobs DJ, Livesay DR (2008) New insight into long-range nonadditivity within protein double-mutant cycles. Proteins 70:915-924

Jarymowycz VA, Stone MJ (2006) Fast time scale dynamics of protein backbones: NMR relaxation methods, applications, and functional consequences. Chem Rev 106:1624-1671

Jensen MO, Borhani DW, Lindorff-Larsen K, Maragakis P, Jogini V, Eastwood MP, Dror RO, Shaw DE (2010) Principles of conduction and hydrophobic gating in $\mathrm{K}+$ channels. Proc Natl Acad Sci USA 107:5833-5838

Jha AK, Colubri A, Freed KF, Sosnick TR (2005) Statistical coil model of the unfolded state: resolving the reconciliation problem. Proc Natl Acad Sci USA 102:13099-13104

Ju T, Bocik W, Majumdar A, Tolman JR (2010) Solution structure and dynamics of human ubiquitin conjugating enzyme Ube2g2. Proteins 78:1291-1301 
Kamerlin SC, Warshel A (2010) At the dawn of the 21st century: is dynamics the missing link for understanding enzyme catalysis? Proteins 78:1339-1375

Kamerzell TJ, Middaugh CR (2007) Two-dimensional correlation spectroscopy reveals coupled immunoglobulin regions of differential flexibility that influence stability. Biochemistry 46:9762-9773

Karplus M (2003) Molecular dynamics of biological macromolecules: a brief history and perspective. Biopolymers $68: 350-358$

Karplus M (2010) Role of conformation transitions in adenylate kinase. Proc Natl Acad Sci USA 107:E71 (author reply E72)

Kay LE, Torchia DA, Bax A (1989) Backbone dynamics of proteins as studied by $15 \mathrm{~N}$ inverse detected heteronuclear NMR spectroscopy: application to staphylococcal nuclease. Biochemistry 28:8972-8979

Kendrew JC, Bodo G, Dintzis HM, Parrish RG, Wyckoff H, Phillips DC (1958) A three-dimensional model of the myoglobin molecule obtained by X-ray analysis. Nature 181:662-666

Kneller GR, Smith JC (1994) Liquid-like side-chain dynamics in myoglobin. J Mol Biol 242:181-185

Korzhnev DM, Religa TL, Banachewicz W, Fersht AR, Kay LE (2010) A transient and low-populated protein-folding intermediate at atomic resolution. Science 329:1312-1316

Koshland DE (1958) Application of a theory of enzyme specificity to protein synthesis. Proc Natl Acad Sci USA 44:98-104

Koshland DEJ, Nemethy G, Filmer D (1966) Comparison of experimental binding data and theoretical models in proteins containing subunits. Biochemistry 5:365-385

Kumar S, Ma B, Tsai CJ, Sinha N, Nussinov R (2000) Folding and binding cascades: dynamic landscapes and population shifts. Protein Sci 9:10-19

Lakomek NA, Carlomagno T, Becker S, Griesinger C, Meiler J (2006) A thorough dynamic interpretation of residual dipolar couplings in ubiquitin. J Biomol NMR 34:101-115

Lane AN (2001) Nuclear magnetic resonance studies of drug-DNA complexes in solution. Methods Enzymol 340:252-281

Lange OF, Grubmuller H, de Groot BL (2005) Molecular dynamics simulations of protein $\mathrm{G}$ challenge NMR-derived correlated backbone motions. Angew Chem Int Ed Engl 44:3394-3399

Lange OF, Schafer LV, Grubmuller H (2006) Flooding in GROMACS: accelerated barrier crossings in molecular dynamics. J Comput Chem 27:1693-1702

Lange OF, Lakomek NA, Fares C, Schroder GF, Walter KF, Becker S, Meiler J, Grubmuller H, Griesinger C, de Groot BL (2008) Recognition dynamics up to microseconds revealed from an RDC-derived ubiquitin ensemble in solution. Science 320:14711475

Lange OF, van der Spoel D, de Groot BL (2010) Scrutinizing molecular mechanics force fields on the submicrosecond timescale with NMR data. Biophys J 99:647-655

Li DW, Brüschweiler R (2009) Certification of molecular dynamics trajectories with NMR chemical shifts. J Phys Chem Lett $1: 246-248$

Li DW, Brüschweiler R (2010) NMR-based protein potentials. Angew Chem Int Ed Engl 49:6778-6780

Li DW, Meng D, Brüschweiler R (2009) Short-range coherence of internal protein dynamics revealed by high-precision in silico study. J Am Chem Soc 131:14610-14611

Lindorff-Larsen K, Best RB, Depristo MA, Dobson CM, Vendruscolo M (2005a) Simultaneous determination of protein structure and dynamics. Nature 433:128-132

Lindorff-Larsen K, Best RB, Vendruscolo M (2005b) Interpreting dynamically-averaged scalar couplings in proteins. $\mathrm{J}$ Biomol NMR 32:273-280

Liu T, Whitten ST, Hilser VJ (2006) Ensemble-based signatures of energy propagation in proteins: a new view of an old phenomenon. Proteins 62:728-738
Long D, Brüschweiler R (2011a) In silico elucidation of the recognition dynamics of ubiquitin. PLoS Comput Biol 7:e1002035

Long D, Brüschweiler R (2011b) Atomistic kinetic model of population shift and allostery in biomolecules. J Am Chem Soc. doi:10.1021/ja208813t

Loria JP, Rance M, Palmer AG (1999) A relaxation-compensated Carr-Purcell-Meiboom-Gill sequence for characterizing chemical exchange by NMR spectroscopy. J Am Chem Soc 121:2331-2332

Ma B, Kumar S, Tsai CJ, Nussinov R (1999) Folding funnels and binding mechanisms. Protein Eng 12:713-720

Ma B, Tsai CJ, Pan Y, Nussinov R (2010) Why does binding of proteins to DNA or proteins to proteins not necessarily spell function? ACS Chem Biol 5:265-272

Markwick PR, Bouvignies G, Salmon L, McCammon JA, Nilges M, Blackledge M (2009) Toward a unified representation of protein structural dynamics in solution. J Am Chem Soc 131:1696816975

Mayer KL, Earley MR, Gupta S, Pichumani K, Regan L, Stone MJ (2003) Covariation of backbone motion throughout a small protein domain. Nat Struct Biol 10:962-965

McCammon JA, Gelin BR, Karplus M (1977) Dynamics of folded proteins. Nature 267:585-590

Meyer T, D'Abramo M, Hospital A, Rueda M, Ferrer-Costa C, Perez A, Carrillo O, Camps J, Fenollosa C, Repchevsky D, Gelpi JL, Orozco M (2010) MoDEL (Molecular Dynamics Extended Library): a database of atomistic molecular dynamics trajectories. Structure 18:1399-1409

Missimer JH, Dolenc J, Steinmetz MO, van Gunsteren WF (2010) Exploring the trigger sequence of the GCN4 coiled-coil: biased molecular dynamics resolves apparent inconsistencies in NMR measurements. Protein Sci 19:2462-2474

Mittermaier A, Kay LE (2006) New tools provide new insights in NMR studies of protein dynamics. Science 312:224-228

Monod J, Wyman J, Changeux JP (1965) On the nature of allosteric transitions: a plausible model. J Mol Biol 12:88-118

Muirhead H, Perutz MF (1963) Structure of haemoglobin. A threedimensional Fourier synthesis of reduced human haemoglobin at $5.5 \AA$ resolution. Nature 199:633-638

Nechushtai R, Lammert H, Michaeli D, Eisenberg-Domovich Y, Zuris JA, Luca MA, Capraro DT, Fish A, Shimshon O, Roy M, Schug A, Whitford PC, Livnah O, Onuchic JN, Jennings PA (2011) Allostery in the ferredoxin protein motif does not involve a conformational switch. Proc Natl Acad Sci USA 108: $2240-2245$

Nodet G, Salmon L, Ozenne V, Meier S, Jensen MR, Blackledge M (2009) Quantitative description of backbone conformational sampling of unfolded proteins at amino acid resolution from NMR residual dipolar couplings. J Am Chem Soc 131:1790817918

Okazaki K, Takada S (2008) Dynamic energy landscape view of coupled binding and protein conformational change: induced-fit versus population-shift mechanisms. Proc Natl Acad Sci USA 105:11182-11187

Olsson U, Wolf-Watz M (2010) Overlap between folding and functional energy landscapes for adenylate kinase conformational change. Nat Commun 1:111

Pai EF, Sachsenheimer W, Schirmer RH, Schulz GE (1977) Substrate positions and induced-fit in crystalline adenylate kinase. J Mol Biol 114:37-45

Palmer AG, Kroenke CD, Loria JP (2001) Nuclear magnetic resonance methods for quantifying microsecond-to-millisecond motions in biological macromolecules. Methods Enzymol 339:204-238

Pan Y, Tsai CJ, Ma B, Nussinov R (2009) How do transcription factors select specific binding sites in the genome? Nat Struct Mol Biol 16:1118-1120 
Pan Y, Tsai CJ, Ma B, Nussinov R (2010) Mechanisms of transcription factor selectivity. Trends Genet 26:75-83

Perez A, Luque FJ, Orozco M (2007a) Dynamics of B-DNA on the microsecond time scale. J Am Chem Soc 129:14739-14745

Perez A, Marchan I, Svozil D, Sponer J, Cheatham TE, Laughton CA, Orozco M (2007b) Refinement of the AMBER force field for nucleic acids: improving the description of alpha/gamma conformers. Biophys J 92:3817-3829

Qin F, Chen Y, Li YX, Chen HF (2009) Induced fit for mRNA/ TIS11d complex. J Chem Phys 131:115103

Rajagopalan PT, Lutz S, Benkovic SJ (2002) Coupling interactions of distal residues enhance dihydrofolate reductase catalysis: mutational effects on hydride transfer rates. Biochemistry 41:1261812628

Ramirez UD, Focia PJ, Freymann DM (2008) Nucleotide-binding flexibility in ultrahigh-resolution structures of the SRP GTPase Ffh. Acta Crystallogr D Biol Crystallogr 64:1043-1053

Richter B, Gsponer J, Varnai P, Salvatella X, Vendruscolo M (2007) The MUMO (minimal under-restraining minimal over-restraining) method for the determination of native state ensembles of proteins. J Biomol NMR 37:117-135

Rod TH, Radkiewicz JL, Brooks CL (2003) Correlated motion and the effect of distal mutations in dihydrofolate reductase. Proc Natl Acad Sci USA 100:6980-6985

Ryabov Y, Fushman D (2006) Interdomain mobility in di-ubiquitin revealed by NMR. Proteins 63:787-796

Salmon L, Nodet G, Ozenne V, Yin G, Jensen MR, Zweckstetter M, Blackledge M (2010) NMR characterization of long-range order in intrinsically disordered proteins. J Am Chem Soc 132:84078418

Salmon L, Bouvignies G, Markwick P, Blackledge M (2011) Nuclear magnetic resonance provides a quantitative description of protein conformational flexibility on physiologically important time scales. Biochemistry 50:2735-2747

Salsas-Escat R, Stultz CM (2010) Conformational selection and collagenolysis in type III collagen. Proteins 78:325-335

Schuetz P, Wuttke R, Schuler B, Caflisch A (2010) Free energy surfaces from single-distance information. J Phys Chem B 114:15227-15235

Schwieters CD, Clore GM (2007) A physical picture of atomic motions within the Dickerson DNA dodecamer in solution derived from joint ensemble refinement against NMR and largeangle X-ray scattering data. Biochemistry 46:1152-1166

Scott WR, Mark AE, van Gunsteren WF (1998) On using timeaveraging restraints in molecular dynamics simulation. J Biomol NMR 12:501-508

Searle MS, Embrey KJ (1990) Sequence-specific interaction of Hoechst 33258 with the minor groove of an adenine-tract DNA duplex studied in solution by $1 \mathrm{H}$ NMR spectroscopy. Nucleic Acids Res 18:3753-3762

Shapiro YE, Kahana E, Tugarinov V, Liang Z, Freed JH, Meirovitch E (2002) Domain flexibility in ligand-free and inhibitor-bound Escherichia coli adenylate kinase based on a mode-coupling analysis of $15 \mathrm{~N}$ spin relaxation. Biochemistry 41:6271-6281

Shaw DE, Maragakis P, Lindorff-Larsen K, Piana S, Dror RO, Eastwood MP, Bank JA, Jumper JM, Salmon JK, Shan Y, Wriggers W (2010) Atomic-level characterization of the structural dynamics of proteins. Science 330:341-346

Showalter SA, Brüschweiler R (2007) Quantitative molecular ensemble interpretation of NMR dipolar couplings without restraints. J Am Chem Soc 129:4158-4159

Showalter SA, Brüschweiler R (2007) Validation of molecular dynamics simulations of biomolecules using NMR spin relaxation as benchmarks: application to the AMBER99SB force field. J Chem Theory Comput 3:961-975
Stelzer AC, Kratz JD, Zhang Q, Al-Hashimi HM (2010) RNA dynamics by design: biasing ensembles towards the ligandbound state. Angew Chem Int Ed Engl 49:5731-5733

Stelzer AC, Frank AT, Kratz JD, Swanson MD, Gonzalez-Hernandez MJ, Lee J, Andricioaei I, Markovitz DM, Al-Hashimi HM (2011) Discovery of selective bioactive small molecules by targeting an RNA dynamic ensemble. Nat Chem Biol 7:553-559

Straub FB, Szabolcsi G (1964) Remarks on the dynamic aspect of enzyme structure. In: Braunstein AE (ed) Molecular biology: problems and perspectives. Nauka, Moscow, pp 182-187

Theobald DL, Wuttke DS (2008) Accurate structural correlations from maximum likelihood superpositions. PLoS Comput Biol 4:e43

Tonelli M, Ulyanov NB, Billeci TM, Karwowski B, Guga P, Stec WJ, James TL (2003) Dynamic NMR structures of [Rp]- and [Sp]phosphorothioated DNA-RNA hybrids: is flexibility required for RNase H recognition? Biophys J 85:2525-2538

Torda AE, Scheek RM, van Gunsteren WF (1989) Time averaged distance restraints in molecular dynamics simulations. Chem Phys Lett 157:289-294

Tsai CJ, Kumar S, Ma B, Nussinov R (1999a) Folding funnels, binding funnels, and protein function. Protein Sci 8:1181-1190

Tsai CJ, Ma B, Nussinov R (1999b) Folding and binding cascades: shifts in energy landscapes. Proc Natl Acad Sci USA 96:99709972

Tsai CJ, del Sol A, Nussinov R (2008) Allostery: absence of a change in shape does not imply that allostery is not at play. J Mol Biol 378:1-11

Tsai CJ, Del Sol A, Nussinov R (2009) Protein allostery, signal transmission and dynamics: a classification scheme of allosteric mechanisms. Mol Biosyst 5:207-216

van Gunsteren WF, Dolenc J (2008) Biomolecular simulation: historical picture and future perspectives. Biochem Soc Trans 36:11-15

Vertessy BG, Orosz F (2011) From "fluctuation fit" to "conformational selection": evolution, rediscovery, and integration of a concept. Bioessays 33:30-34

Viswanathan M, Linthicum DS, Subramaniam S (2000) Analysis of correlated motion in antibody combining sites from molecular dynamics simulations. Methods 20:362-371

Vogeli B, Yao L, Bax A (2008) Protein backbone motions viewed by intraresidue and sequential $\mathrm{HN}-\mathrm{Halpha}$ residual dipolar couplings. J Biomol NMR 41:17-28

Watson JD, Crick FH (1953) Molecular structure of nucleic acids; a structure for deoxyribose nucleic acid. Nature 171:737-738

Whitley MJ, Lee AL (2009) Frameworks for understanding longrange intra-protein communication. Curr Protein Pept Sci 10:116-127

Williamson JR (2000) Induced fit in RNA-protein recognition. Nat Struct Biol 7:834-837

Wlodarski T, Zagrovic B (2009) Conformational selection and induced fit mechanism underlie specificity in noncovalent interactions with ubiquitin. Proc Natl Acad Sci USA 106: 19346-19351

Zhang Q, Sun X, Watt ED, Al-Hashimi HM (2006) Resolving the motional modes that code for RNA adaptation. Science 311:653-656

Zhang Q, Stelzer AC, Fisher CK, Al-Hashimi HM (2007) Visualizing spatially correlated dynamics that directs RNA conformational transitions. Nature 450:1263-1267

Zheng W (2010) Anharmonic normal mode analysis of elastic network model improves the modeling of atomic fluctuations in protein crystal structures. Biophys J 98:3025-3034

Zhou HX (2010) From induced fit to conformational selection: a continuum of binding mechanism controlled by the timescale of conformational transitions. Biophys J 98:L15-L17 